\title{
An Analytical Approach in Calculation of the inductance of Single-Phase Asynchronous Motors
}

\author{
Rusdy Hartungi, Luis Gomez-Agustina, and Zhihui Ye
}

\begin{abstract}
In optimizing design of the iron core, it is essential to know the magnetic field of the coil outside the core to get the leakage inductance, because the leakage inductance in this area usually provides high contribution to the flux density in the iron core. In this paper, an analytical solution as an alternative to three-dimensional FEM (finite element method) to calculate the field outside the iron core by solving analytical equation using MathCAD is presented. The analytical method provides results that are close to result given by three-dimensional FEM, but with the great advantage of dramatically reduced computing time.
\end{abstract}

Index Terms - Inductance, Single Phase Motors, AC motors.

\section{INTRODUCTION}

$\mathrm{T}$ here are many applications, where a concentrated winding around an iron core is used. A good example is the single-phase two-pole permanent magnet motor that could also work as an actuator. This motor is found in many household appliances and pumps, but as actuators they are used as a direct drive of the throttle plate for internal combustion engines in the automobile industry. The construction of such motor or actuator is shown in Fig. 1. It is normally found that rather sophisticated design tools or software programs for calculation of torques and power of electric machines are available, however often these tools and software have major drawbacks, as they ignore the leakage flux outside the iron core, the flux density level of the iron (i.e. the saturation level) or the leakage inductance are not calculated precisely.

Manuscript received January 3, 2020, revised January 9 and January 13 , 2020 , accepted January $14,2020$.

Rusdy Hartungi is with School of Built Envirinment and Architecture, Civil and Building Services Engineering, London South Bank University, United Kingdom (e-mail: hartungr@1sbu.ac.uk).

Luis Gomez-Agustina is a Senior Lecturer and active researcher in Energy, Mechanical and Electrical System in Building at London South Bank University (e-mail: gomezagl@1sbu.ac.uk).

Zhihui Ye is a Senior Lecturer in the School of Built Environment and Architecture, London South Bank University, United Kingdom (e-mail: zhihui.ye@1sbu.ac.uk).

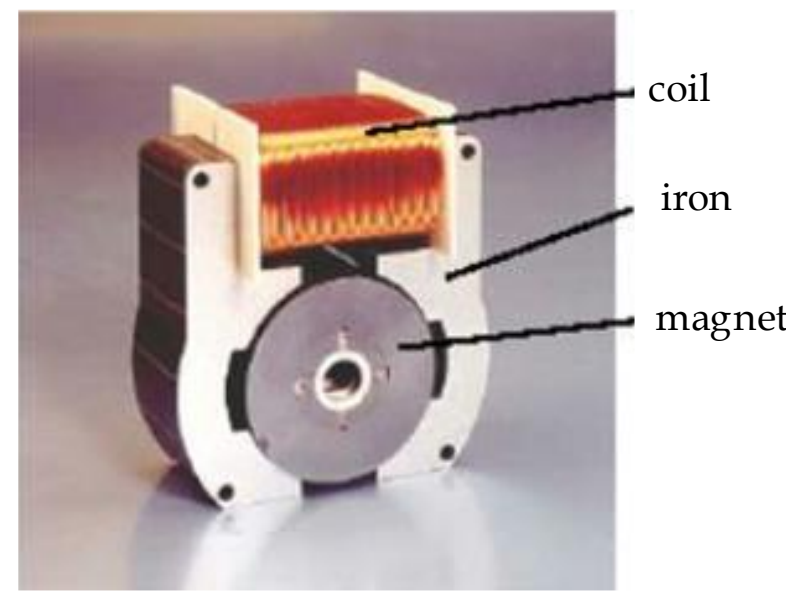

Fig. 1. Single Phase Permanent Magnet Motor/Actuator

As the coil is wound around the iron core, such as in a singlephase permanent magnet motor/actuator, as shown in section $\mathrm{BB}$ in Fig. 2, the leakage flux is rather high, therefore it is rather imprecise to ignore them.

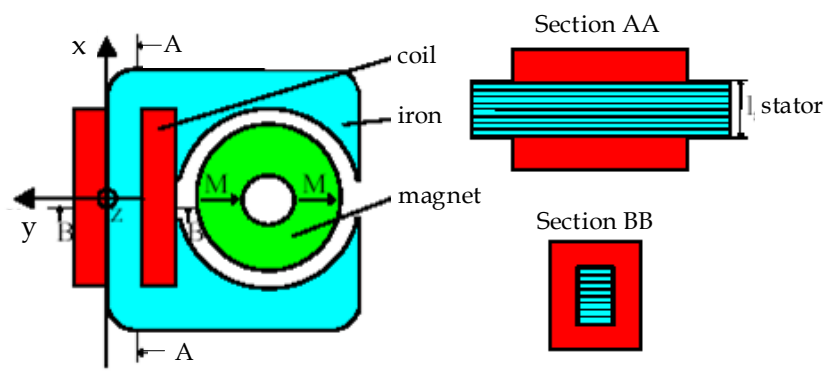

Fig. 2. Construction of single-phase two-pole permanent magnet motor.

Thus, it is essential that in a good motor design and calculation of leakage inductance to incorporate the leakage field in this area. Neglecting this leakage inductance provides under estimates of leakage inductance; motor saturates at low current and therefore, the real output torque is much lower that estimated. Up to now this problem can only be solved using three-dimensional Finite Element Method (FEM). Two-dimensional FEM-calculations will give wrong results, because the large coils overhang in axial direction of the magnet is neglected. The problem with three-dimensional FEM is that three-dimensional FEM are rather costly as it takes much computation time as well as need pre- and postprocessing procedure. Therefore this FEM method is impractical to be included into a design procedure. Because of this an alternative using analytical approach will be presented here.

To avoid an iterative calculation of the flux density in the iron core, in this analytical method the relative permeability 
of the iron is assumed to be infinite $\left(\mu_{r, F e} \rightarrow \infty\right)$. This means that, this analytical method assume that there is no saturation exist (a normal approach in solving leakage flux using analytical method [1].

The assumption of infinite relative permeability of the stator iron has the following consequences in using this approach in practice:

- The flux density level of the iron core calculated by this procedure has to be checked against the material data: If the calculated value is above the saturation level of the material, some input data have to be changed to remain below the saturation level of the material.

- A single value of the coil inductivity will be given. As the inductivity decreases with increasing saturation, the coil inductivity is constant below the saturation value.

\section{MAGNETIC FIELD CALCULATION}

The Cartesian coordinate system that will be used in this model is defined as shown in Fig. 2. In this model we will restrict the calculation of the magnetic field to values $y \geq 0$ in the $x-y$ plane. Additional contributions to the flux density in the iron core come from the part of the coil in the $x-z$ plane. As the method of calculation is in principle the same, this will not be described in detail. With this, the total threedimensional field problem is divided into several twodimensional ones. Separation of the flux density in several contributions and adding these up is allowed because of the linearity of the problem (the assumption of infinite relative permeability of the stator iron was made, see above).

\section{A. Derivation of an Equivalent Problem}

To find solution we are regarding the following twodimensional problem: instead of considering the outside radius of the stacked iron, a lamination with rectangular outside contour is assumed [2, 3]. In addition, the case of a coil facing a finite iron will be approximated by the case of a coil facing an infinite iron half plane [4]. In the half plane $y \geq$ 0 this approximated field problem is equivalent to that one we get with the help of reflection method as shown in Fig. 3. The Cartesian coordinate system is defined as shown in Fig. 2, because using this coordinate system the field distribution in which we are interested will be represented in the first quadrant $(x \geq 0, y \geq 0)$. This makes the graphical representation easier when the software tool MathCAD [5, 6] is used for the calculation of the field distribution.

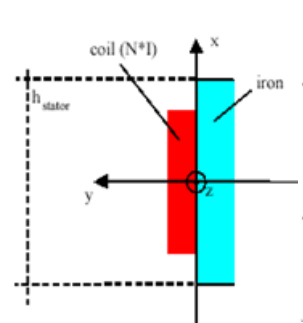

(a)

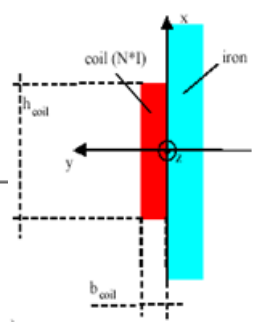

(b)

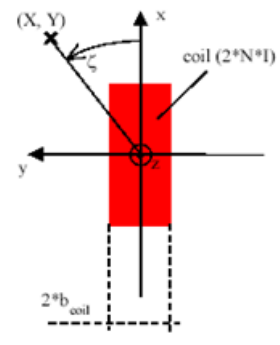

(c)
Fig. 3. (a). 2-dimensional Finite Problem; (b). Equivalent of 2-dimensional Finite Problem (infinite iron half plane); (c). Reflection Method

The field problem described above can now be solved using Ampere's law:

$$
\oint \vec{H} \cdot \overrightarrow{d l}=N . I
$$

To get an impression which degree of simplification is permissible, the following three calculations will be performed:

- concentrating the coil to a single point in the $x$-y plane at $x=y=0$ (i.e. "0-dimensional current distribution")

- concentrating the coil to a line segment $(y=0$, $-\frac{1}{2} h_{\text {coil }} \leq x \leq+\frac{1}{2} h_{\text {coil }}$ )

- regarding the real two-dimensional coil (

$$
\left.-b_{\text {coil }} \leq y \leq+b_{\text {coil }},-\frac{1}{2} h_{\text {coil }} \leq x \leq+\frac{1}{2} h_{\text {coil }}\right)
$$

In the last two calculations the total current (2.NI) has to be distributed equally on the line segment or area, so that a surface current density or a current density, respectively, has to be considered. The solution of Equation (1) will then be got through integration (over line segment or area, respectively). In the following, the positive direction of the current flow is assumed to be in the positive direction of the $z$-axis.

If the coil is concentrated to a single point, the solution of the field problem will not be cumbersome: The flux lines are concentric circles around the coil, the field strength decreases inversely proportional to the distance from the coil. As we are calculating for air permeability in the whole plane (see Fig. 3c) the flux density is $\vec{B}=\mu_{0} \vec{H}$. In the last two calculations the total current (2.NI) has to be distributed equally on the line segment or area, so that a surface current density or a current density, respectively, has to be considered. The solution of Equation (1) will then be got through integration (over line segment or area, respectively). In the following, the positive direction of the current flow is assumed to be in the positive direction of the z-axis. If the coil is concentrated to a single point, the solution of the field problem will not be cumbersome: The flux lines are concentric circles around the coil, the field strength decreases inversely proportional to the distance from the coil.

\section{B. Calculation for the 0-dimensional current distribution}

For a specific point $(X, Y)$ of the $x-y$ plane the $x$ and $y$ components of the flux density distribution become (the index "0" stands for the 0-dimensional current distribution):

$$
\begin{aligned}
& B_{0, x}(X, Y)=\frac{\mu_{0}}{2 \pi} 2 N I \frac{-\sin (\zeta)}{\sqrt{X^{2}+Y^{2}}} \quad(X, Y) \neq(0,0) \\
& B_{0, y}(X, Y)=\frac{\mu_{0}}{2 \pi} 2 N I \frac{-\cos (\zeta)}{\sqrt{X^{2}+Y^{2}}} \quad(X, Y) \neq(0,0)
\end{aligned}
$$

For $(X, Y)=(0,0)$ the field strength is not defined as the value for $\overrightarrow{d l}$ in Equation (1) will be zero. The angle $\zeta$ can be calculated from $\zeta=\arctan \left(\frac{Y}{X}\right)$.

\section{Calculation for the one-dimensional current distribution}

If the coil is concentrated to a line segment $(y=0$, $\left.-\frac{1}{2} h_{\text {coil }} \leq x \leq+\frac{1}{2} h_{\text {coil }}\right)$, the $\mathrm{x}$ and $\mathrm{y}$ components of the flux density distribution for a specific point), $(X, Y)$ can be calculated as follows (the index "1" stands for the 1dimensional current distribution): 


$$
\begin{gathered}
B_{1, x}(X, Y)=\frac{\mu_{0}}{2 \pi} \frac{2 N I}{h_{\text {coil }}} \int_{-\frac{1}{2} h_{\text {coil }}}^{\frac{1}{2} h_{\text {coil }}} \frac{-\sin (\zeta)}{\sqrt{(X-x)^{2}+Y^{2}}} d x \\
(X-x, Y) \neq(0,0) \\
B_{1, y}(X, Y)=\frac{\mu_{0}}{2 \pi} \frac{2 N I}{h_{\text {coil }}} \int_{-\frac{1}{2} h_{\text {coil }}}^{\frac{1}{2} h_{\text {coil }}} \frac{\cos (\zeta)}{\sqrt{(X-x)^{2}+Y^{2}}} d x \\
(X-x, Y) \neq(0,0)
\end{gathered}
$$

The angle $\zeta$ can be calculated from $\zeta=\arctan \left(\frac{Y}{X-x}\right)$

\section{Calculation for the two-dimensional current distribution}

Considering the coil in its real two-dimensional expansion ( $\left.-b_{\text {coil }} \leq y \leq+b_{\text {coil }}, \quad-\frac{1}{2} h_{\text {coil }} \leq x \leq+\frac{1}{2} h_{\text {coil }}\right)$ the $x$ and $y$ components of the flux density distribution for a specific point $(X, Y)$ can be calculated as follows (the index " 2 " stands for the two-dimensional current distribution):

$$
\begin{aligned}
& B_{2, x}(X, Y)= \\
& \frac{\mu_{0}}{2 \pi} \frac{2 N I}{h_{\text {coil }} 2 b_{\text {coil }}} \int_{-\frac{1}{2} h_{\text {coil }}}^{\frac{1}{2} h_{\text {coil }}} \int_{b_{\text {coil }}}^{b_{\text {coil }}} \frac{-\sin (\zeta)}{\sqrt{(X-x)^{2}+(Y-y)^{2}}} d x d y \\
& \quad(X-x, Y-y) \neq(0,0) \\
& B_{2, y}(X, Y)= \\
& \frac{\mu_{0}}{2 \pi} \frac{2 N I}{h_{\text {coil }} 2 b_{\text {coil }}} \int_{-\frac{1}{2} h_{\text {coil }}}^{\frac{1}{2} h_{\text {coil }}} \int_{b_{\text {coil }}}^{b_{\text {coil }}} \frac{\cos (\zeta)}{\sqrt{(X-x)^{2}+(Y-y)^{2}}} d x d y \\
& \quad(X-x, Y-y) \neq(0,0)
\end{aligned}
$$

The angle $\zeta$ can be calculated from $\zeta=\arctan \left(\frac{Y-y}{X-x}\right)$

\section{E. Numerical Calculation}

To solve Equations $(2 \mathrm{a}, 2 \mathrm{~b})$ to $(4 \mathrm{a}, 4 \mathrm{~b})$ the "MathCAD" program has been used, where using this program, the integrals in equation $(3 a, 3 b)$ and $(4 a, 4 b)$ are solved numerically. In Figs. 4 and 5 it is shown the flux density distribution for the first quadrant $(x \geq 0, y \geq 0)$ for one- and two-dimensional current distribution.

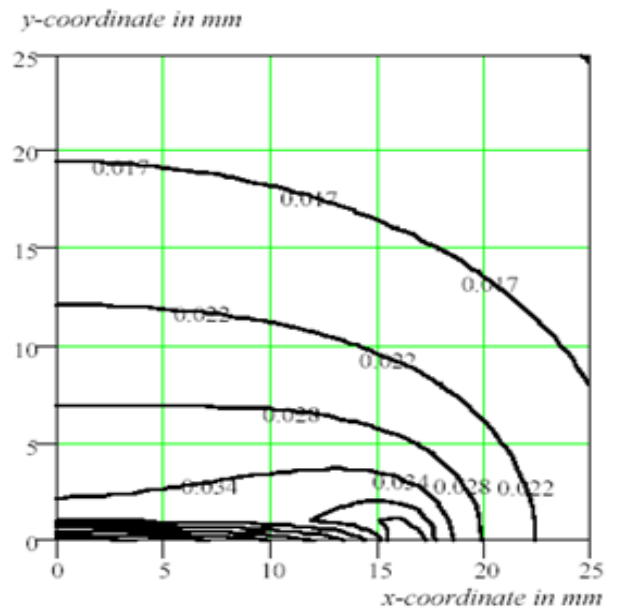

Fig. 4. Distribution of magnetic flux density (in $\mathrm{T}$ ) for the 1-dimensional current distribution

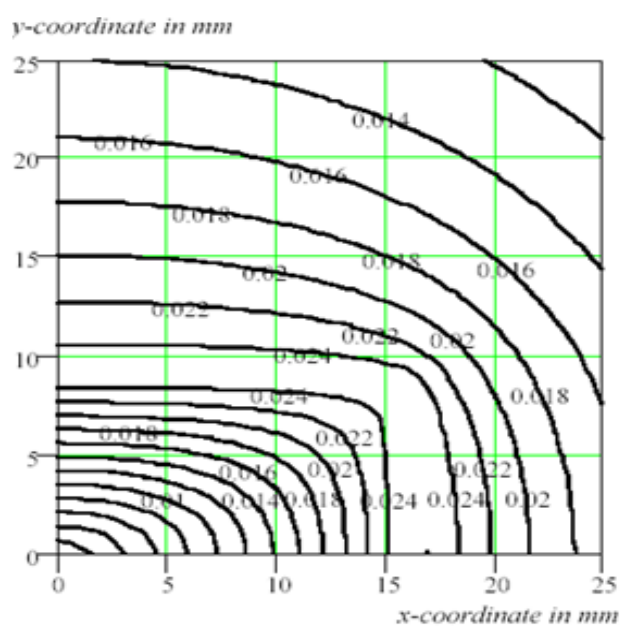

Fig. 5. Distribution of magnetic flux density (in T) for the 2-dimensional current distribution

The $x$-axis (Fig. 2) is represented vertically while the $y$ axis horizontally. The calculation has been performed for $N I$ $=1000 \mathrm{~A}, h_{\text {coil }}=33.10^{-3} \mathrm{~m}$ and $b_{\text {coil }}=9.10^{-3} \mathrm{~m}$. There is no field plot for the 0-dimensional current distribution (i.e. current concentrated in the center of the coordinate system), because the result is obvious: We get concentric circles around $x=y=0$ with e.g. $B_{0} \mid=40 \mathrm{mT}$ for a radius of $10 \mathrm{~mm}$. It is obvious from these calculations, that only calculation of the two-dimensional current distribution gives the right result. For evaluating the flux density in the iron core of the coil and the inductivity of the coil, it is sufficient to know the flux density on the iron surface, i.e. the flux density for $y=0$. In addition, only the y component has to be calculated: The $x$ component is always equal to zero, because it is assumed that $\mu_{r, F e} \rightarrow \infty$ (the flux density vector is always perpendicular to the iron surface if $\mu_{r, F e} \rightarrow \infty$ is true). Because of this, the complexity of calculation and time needed for iteration can be reduced. In Fig. 6 it can be inferred that even for calculating the flux density on the iron surface it is still needed to consider the two-dimensional current distribution to obtain the correct result.

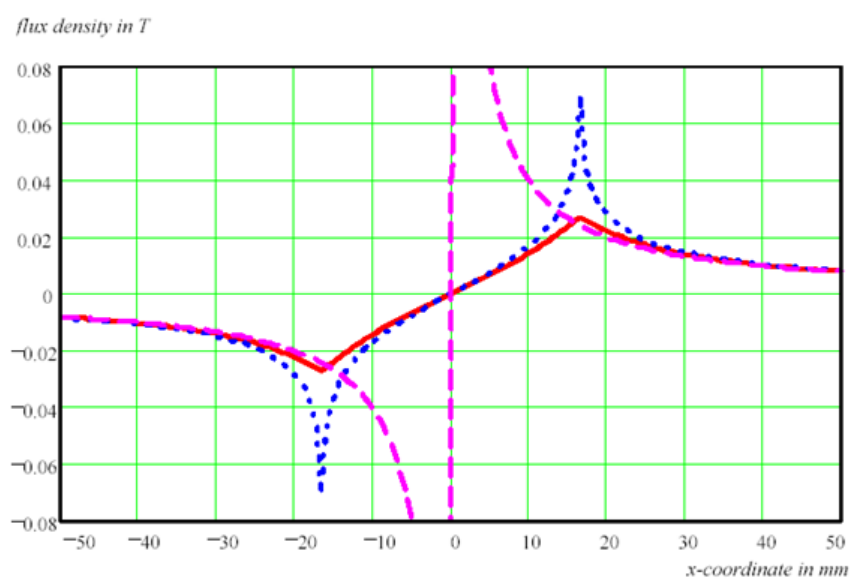

Fig. 6: Flux density versus $\mathrm{x}$-coordinate for $0=\mathrm{y}$ and for $0-, 1$ - and twodimensional current distribution (dashed, dotted and solid lines, respectively).

\section{PRACTICAL RESUltS}

To further reduce the complexity and the calculation time, the following simplifications will be introduced: 
- For $|x| \leq \frac{1}{2} h_{\text {stator }}$ (Fig. 3 for the definition of $h_{\text {stator }}$ ) we do calculation for the two-dimensional current distribution (even if the real field distribution will be deformed a little bit by the finite expansion of the stator iron).

- For $|x|>\frac{1}{2} h_{\text {stator }}$ we assume the current to be concentrated into one point at $(x, y)=(0,0)$.

The second assumption is motivated by the fact that for $y=0$ $|x|>\frac{1}{2} h_{\text {stator }}$ the flux density calculated for 0 -dimensional current distribution is a reasonable approximation for the flux density calculated for the two-dimensional current distribution. For example, on the above calculation we get a relatively small error of about $7 \%\left(h_{\text {stator }}=49.10^{-3} \mathrm{~m}\right)$.

With this simplification a numerical evaluation of the motor shown in Fig. 1 has been carried out. It could be shown that the flux density in the iron core coming from the coil leakage is the same magnitude as the flux density coming from the desired flux path (i.e. the flux path crossing the rotary magnet). This means that for an orientation of the rotary magnet like shown in Fig. 2 the flux density of the iron core is doubled just because of the leakage. Evaluating the flux density in the iron core with maximum contribution of the magnet (i.e. a magnet position perpendicular to what is shown in Fig. 2) results in about 25\% of the flux in the iron core coming from leakage.

The inductivity can be calculated by differentiation of the flux linkage with respect to the current:

$$
L=\frac{\partial \Psi}{\partial I}
$$

The flux linkage can be obtained from:

$$
\Psi=N \Phi \quad \Phi=\int_{A}^{B} B \cdot d A
$$

As we assumed that no saturation occurs in the stator iron, the flux density generated by the permanent magnet has no influence on the derivative in Equation (5). Because of the linearity assumption, Equations (5) and (6) can be simplified to be $L=N B A / I$. In addition, we will calculate the inductance just from the flux density in the iron core and the iron core cross section:

$$
L=\frac{N B_{\text {Core }} A_{\text {core }}}{I}
$$

Here, the coil area in calculation of the leakage inductance is neglected since the flux density in this area is relatively small, at least 50 times smaller than in the iron core and that not all field lines are coupled to all turns of the coil (the cross sections of iron core and coil are of the same order of magnitude).

For the purpose of comparison herewith we summarized several methods in calculating leakage inductance using several methods as shown in Table I.

TABLE I. COMPARISON OF CALCULATION OF THE LEAKAGE INDUCTANCE USING SEVERAL METHODS [7]

\begin{tabular}{lcc}
\hline & $\begin{array}{c}\mathrm{L} \\
(m \mathrm{H})\end{array}$ & $\begin{array}{l}\text { Difference with } \\
\text { measurement } \\
\text { Result }(\%)\end{array}$ \\
\hline Analytical calculation without leakage & 6.5 & -58.6 \\
Analytical calculation with leakage & 14.6 & -7.0 \\
3D FEM calculation & 13.6 & 13.4 \\
Measurement & 15.7 & - \\
\hline
\end{tabular}

From Table I, it can be seen that the analytical calculation by considering the leakage provides results that close to the measuring value. The three-dimensional FEM calculation comes next. It is obvious that the calculation without taking into account the leakage leads to wrong results.

\section{CONCLUSIONS}

In this paper an analytical quasi three-dimensional calculation of leakage and inductivity for motors with concentrated windings is presented.

To solve leakage flux problem in analytical manner, the linearity assumption has been made; the three-dimensional problem is then arranged into several two-dimensional. It is obvious from this paper that calculating the exact twodimensional field distribution in each plane is essential in order to know the leakage flux, which itself is important for the flux density level in the iron core as well as for the inductance.

The analytical method provides results that are close to result given by three-dimensional FEM, but with the great advantage of dramatically reduced computing time. There be more advantage could be gained, when small changes are needed to be made on the design. For FEM method the whole model has to be set up again, whereas for the analytical method just needed the adjustment of some input parameters.

\section{REFERENCES}

[1] Muller, Germar and Karl Vogt, "Berechnung elektrischer Maschinen (Elektrische Maschine) (German Edition) Kindle Edition”, Weinheim; Basel [etc.]: VCH, cop., 2018.

[2] Murthy, Vishnu. "Computer Aided Design of Electrical Machines", 1st Edition, Amazon Kindle Edition, 2019.

[3] Rosu, Marius, Ping Zhou, Dingsheng Lin, Dan M. Ionel, Mircea Popescu Frede Blaabjerg, Vandana Rallabandi, David Staton, "Multiphysics Simulation by Design for Electrical Machines, Power Electronics and Drives", IEEE Press Series on Power Engineering, IEEE Press 9 Mar 2018.

[4] Simonyi, Karoli, Heinrich Theil, "Theoretische Elektrotechnik", VEB Verlag der Wissenschaften, Berlin, 1993.

[5] MSC/ARIES, Version 7, The MacNeal-Schwendler Corporation, April 1996.

[6] MathCAD, Prime 6. By PTXC Math CAD 2018/2019.

[7] Hartungi, R, "Modelling and Simulation of Synchronous Machine onLoad under Voltage Sink", Jurnal Teknologi Industri, Vol. VIII, No. 1, January, 2004, pp.73-83.

\section{BIOGRAPHY}

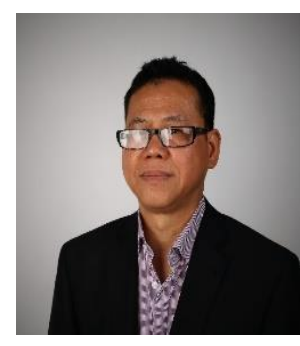

Eur Ing, Dr. Rusdy Hartungi, M.Sc, MBA, CEng MCIBSE, MIET, Int(PE) has a solid research interest in Power Quality and Sustainability. Prior to his current appointment as an academic and researcher at London South Bank University, and previously at the University of Central Lancashire, he was working for many years as a Consulting Engineer working for Multi-National Engineering Company in the UK. Dr. Hartungi is an active member of the Institution of Electrical Engineer (IEE/IET) and Chartered Institution of Building Services Engineers (CIBSE). He is one of a few the UK chartered engineers who have gained international recognition as International Professional Engineers, IntPE(UK) as well as European Professional Engineer (Eur Ing). Dr. 
Hartungi has published more than 40 articles including journals and International conference proceedings.

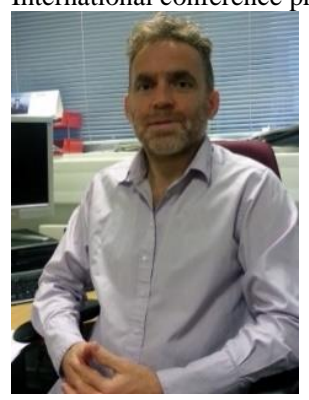

Dr. Luis Gomez-Agustina, MSc, is a Senior Lecturer and active researcher in Energy, Mechanical and Electrical System in Building at London South Bank University (LSBU) in the United Kingdom (UK). He holds a BEng (Hons) in Telecommunication Engineering from Madrid Polytechnic University (Spain), a MSc in Environmental and Architectural Acoustics and PhD in Acoustics from LSBU (UK). Luis has extensive professional experience gained in research and development positions in industrial product design engineering and communication engineering industries. He is an active member of the Institute of Acoustics (UK) and Acoustical Society of America (ASA). He has over 30 national / international conferences and peer reviewed publications.
Luis is currently supervising three $\mathrm{PhD}$ programmes at LSBU. He is reviewer of four esteemed peer reviewed international journals. Currently his research focuses on optimizing electrically powered sound sources.

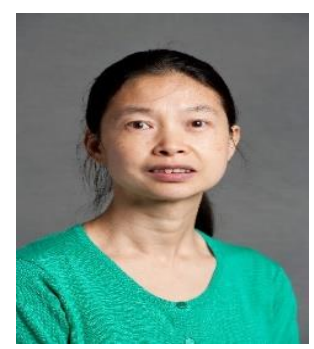

Dr. Zhihui Ye, BEng, M.Sc, MEI, FHEA, is a Senior Lecturer in the School of Built Environment and Architecture, London South Bank University, United Kingdom. Her research interest is in building energy application, the indoor environment and the operational performance of buildings. She is a building service engineer with an MSc in Energy Management and has completed her $\mathrm{PhD}$ in Building Science. 